\title{
Choosing wisely? Multiple Sclerosis and Laboratory Screening for Autoimmune Differential Diagnoses
}

\author{
Do the Guideline Recommendations of the German Society of Neurology Make \\ Sense?
}

\section{(ㄷ) (i) ()ㅜ $\ominus$}

Authors

Jana Becker*, Mareike Geffken*, Rolf R. Diehl, Peter Berlit, Markus Krämer

\author{
Affiliation \\ Alfried Krupp Hospital Essen, Department of Neurology and \\ Neurophysiology
}

Key words

multiple sclerosis, differential diagnosis, ANA

\author{
Bibliography \\ DOI https://doi.org/10.1055/s-0043-115429 \\ Neurology International Open 2017; 1: E256-E263 \\ (c) Georg Thieme Verlag KG Stuttgart · New York \\ ISSN 2511-1795
}

\section{Correspondence}

Priv.-Doz. Dr. med. Markus Krämer

Department of Neurology

Alfried Krupp Hospital

Alfried-Krupp-Straße 21

45130 Essen

Germany

markus.kraemer1977@gmx.de

\begin{abstract}
Background A variety of diseases can mimic the presentation of multiple sclerosis (MS). Diagnosing MS requires the exclusion of conditions considered in the differential diagnosis of MS. The guidelines of the German Society of Neurology (DGN) recommend analyzing 13 mandatory and 9 optional laboratory parameters. The mandatory recommendations include antinu-
\end{abstract}

clear antibodies (ANA) as well as antibodies against double-stranded DNA (anti-dsDNA Abs)).

Methods In a cohort of MS outpatients, those patient records were analyzed in which test results for all or at least some of the recommended laboratory parameters were available. In addition to these laboratory parameters, MRI data, family history, clinical presentation, evoked potentials, and CSF findings were analyzed. Furthermore, a questionnaire was used to prospectively collect information about rheumatological symptoms relevant to the differential diagnosis of MS.

Results In 197 of the included 554 patients, at least some of the recommended differential diagnostic laboratory parameters were available. In $59.4 \%$ of these patients, ANA titers $\geq$ 1:80 were detected. Neither ANA nor ANCA nor anti-dsDNA abs were able to distinguish between MS and non-MS. In 124 MS patients, $54.8 \%$ had positive ANA titers $\geq 1: 80$. MS patients with these titers suffered more frequently from autoimmune diseases and were more likely to have a family history of MS. Of the remaining 73 patients without MS, $67.1 \%$ were ANA positive. In this patient population, the ANA-positive patients were more frequently diagnosed with psychiatric diseases. In the prospectively surveyed MS patients with an ANA titer $>1: 160$, no rheumatologic disease was diagnosed during the average follow-up period of 1.9 years.

Conclusion In line with the "Choosing Wisely" recommendations of the American College of Rheumatology, mandatory laboratory screening appears to offer no benefit to the initial diagnosis of multiple sclerosis, unless a specific differential diagnosis is to be excluded.

\section{Introduction}

The diagnostic criteria for multiple sclerosis (MS) are based on a combined evaluation of clinical symptoms and MRI findings [1]. The MRI criteria for multiple sclerosis introduced by Swanton et al. take location and distribution as well as configuration, number and contrast behavior of MS lesions into account [2]. In more recent studies, perivenous distribution of multiple sclerosis lesions has been shown to be an additional criterion for MS [3, 4]. In German-speaking countries, cerebrospinal fluid (CSF) findings have traditionally played an important role in establishing the initial diagnosis of MS and are still considered of particular importance in this respect today.

\footnotetext{
* equal first authors
} 
According to the Guidelines for Diagnosis and Treatment of Neurological Diseases by the German Society of Neurology (Deutsche Gesellschaft für Neurologie, DGN) [5], it is required for the initial diagnosis of MS to "excluded chronic infectious diseases (neurosyphilis, borreliosis, HIV infection), connective tissue disorders, vasculitis, and leukodystrophies as well as special forms of inflammatory demyelinating diseases (e. g., neuromyelitis optica (NMO) or acute disseminated encephalomyelitis (ADEM)) which are part of the differential diagnosis of MS."

Other conditions often to be considered in the differential diagnosis of MS include cerebral microangiopathy, white matter lesions in migraine as well as unspecific white matter lesions. Apart from immunological disorders, such as neuropsychiatric systemic lupus erythematosus [6], Behcet's disease [7], NMO spectrum disease, and ADEM, numerous other diseases can also mimic MS, including CADASIL [8] and Fabry's disease [9, 10].

The DGN's Guidelines for Diagnosis and Treatment of Neurological Diseases [5] recommend the following parameters as mandatory laboratory tests in the initial workup of patients with suspected MS:

- CRP

- Complete blood count (CBC)

- Blood serum chemistry

- Blood glucose

- Vitamine B12

- Rheumatoid factor

- ANA

- Anti-phospholipid antibodies

- Anti-dsDNA antibodies

- Lupus anticoagulant

- ACE

- Borrelia serology

- Urine analysis

The guidelines recommend the following laboratory tests on a facultative basis if clinical information supports the possibility of a corresponding differential diagnosis:

- c/p-ANCA

- ENA profile

- Autoantibodies to aquaporin-4

- HIV serology

- HTLV-1 serology

- TPHA

- Long-chain fatty acids

- Mycoplasma serology

- Urinary methylmalonic acid excretion

With no supporting literature references provided in the guidelines, the recommendation to perform these tests is based on expert opinion.

In patients with suspected MS, the DGN guidelines also recommend to "find out about potential previous episodes with neurological symptoms" or "other autoimmune diseases experienced by the patients or their family members" [5]. However, no information about any specific symptom patterns characteristic of the respective differential diagnostic conditions is provided.

Several studies evaluated the significance of antibodies associated with diseases listed in the differential diagnosis of MS. These include ANA, ANCA, antiphospholipid autoantibodies, anti-ß2 glycoprotein, lupus anticoagulant, anti-SS-A and anti-SS-B antibodies, as well as antithyroid peroxidase antibodies. For example, Karussis et al. [11] found anticardiolipin antibodies in 20 of 100 patients with probable or definite diagnosis of MS according to Poser's criteria. This subgroup was characterized by slower disease progression and for MS rather atypical symptoms, such as headache, and a lack of oligoclonal banding in CSF. Assuming a special subgroup of MS with underlying vascular mechanisms, the authors discussed treatment of this condition with antiplatelet or even anticoagulant agents.

By contrast, Heinzlef et al. did not regard MS patients testing positive for anticardiolipin antibodies as a subgroup of MS. Given the concomitant greater frequency of other antibodies in these patients, the authors concluded that anticardiolipin antibody positivity only reflected an increased general autoimmune activation [12].

In 1995, Barned et al. postulated in a paper published in Neurology that increased ANA titers in MS patients are most probably only a sign of autoimmune dysregulation. They found positive ANA titers in $26.7 \%$ of patients with relapsing-remitting MS (RRMS) and $30.4 \%$ of patients with secondary-progressive MS (SPMS), even though these patients did not have systemic lupus erythematosus (SLE) [13]. Collard et al. confirmed these results and supported their hypothesis of immunological dysregulation [14].

Garg et al. showed that one or more autoantibodies (antiphospholipid antibodies, ANA, ANCA, anti-SS-A and anti-SS-B antibodies, antithyroid peroxidase antibodies) were detectable in $69 \%$ of analyzed MS and clinically isolated syndrome (CIS) patients (mostly transient positivity with CIS) and that the presence of antibodies was associated with higher T2-lesion load in MRI [15].

Etemadifar et al. did not find an increased prevalence of autoimmune antibodies in patients with optic neuritis compared with the general population. This led to the recommendation to only perform connective tissue disorders laboratory screening in patients with a first demyelinating attack if they have a previous history of rheumatological disease or present with signs and symptoms indicative of a connective tissue disorder [16].

Clinical experiences in day-to-day patient management raised the following questions:

1) Are the laboratory tests recommended as mandatory in the DGN's Guidelines for Diagnosis and Treatment of Neurological Diseases helpful in differentiating between the presence and absence of MS?

2) Can these laboratory values detect differential diagnostic conditions, especially rheumatological diseases?

3) Based on the listed laboratory values, is it possible to identify differences with regard to type of symptoms, secondary diagnoses, results of diagnostic investigations (MRI and CSF findings), clinical presentation, type of clinical course, and family history in MS patients?

4) Can the mentioned laboratory values predict the development of MS or a rheumatological disorder?

The aim of this study is to evaluate the relevance of the laboratory parameters recommended as mandatory or facultative by the DGN. It shall be assessed whether it makes sense to perform the laboratory tests recommended in the DGN guidelines in all patients with suspected multiple sclerosis [5]. 
- Table 1 Tested and abnormal laboratory parameters.

\begin{tabular}{|c|c|c|c|c|c|c|c|c|c|c|c|c|}
\hline \multirow[t]{3}{*}{ Parameter } & \multicolumn{4}{|c|}{ Total patient population } & \multicolumn{4}{|c|}{ MS patient population } & \multicolumn{4}{|c|}{ Non-MS patient population } \\
\hline & \multicolumn{2}{|c|}{ tested } & \multicolumn{2}{|c|}{ abnormal } & \multicolumn{2}{|c|}{ tested } & \multicolumn{2}{|c|}{ abnormal } & \multicolumn{2}{|c|}{ tested } & \multicolumn{2}{|c|}{ abnormal } \\
\hline & $\mathbf{N}$ & $\%$ & $\mathbf{N}$ & $\%$ & $\mathbf{N}$ & $\%$ & $\mathbf{N}$ & $\%$ & $\mathbf{N}$ & $\%$ & $\mathbf{N}$ & $\%$ \\
\hline Blood count & 191 & 97 & 11 & 5.8 & 120 & 96.8 & 5 & 4.2 & 71 & 97.3 & 6 & 8.5 \\
\hline CRP & 193 & 98 & 20 & 10.4 & 121 & 97.6 & 12 & 9.9 & 72 & 98.6 & 8 & 11.1 \\
\hline Vitamine B12 & 100 & 50.8 & 5 & 5 & 54 & 43.5 & 3 & 5.6 & 46 & 63 & 2 & 4.3 \\
\hline Folic acid & 89 & 45.2 & 6 & 6.7 & 44 & 35.5 & 4 & 9.1 & 45 & 61.6 & 2 & 4.4 \\
\hline MMA & 14 & 7.1 & 0 & 0 & 6 & 4.8 & 0 & 0 & 8 & 11 & 0 & 0 \\
\hline Holo-TC & 15 & 7.6 & 4 & 26.7 & 6 & 4.8 & 3 & 50 & 9 & 12.3 & 1 & 11.1 \\
\hline Rheumatoid factor & 113 & 57.4 & 3 & 2.7 & 73 & 58.9 & 1 & 1.4 & 40 & 54.8 & 2 & 5 \\
\hline ANA & 197 & 100 & 117 & 59.4 & 124 & 100 & 68 & 54.8 & 73 & 100 & 49 & 67.1 \\
\hline anti-dsDNA Abs & 140 & 71.1 & 5 & 3.6 & 82 & 66.1 & 3 & 3.7 & 58 & 79.5 & 2 & 3.4 \\
\hline ENA & 183 & 92.9 & 5 & 2.7 & 114 & 91.9 & 4 & 3.5 & 69 & 94.5 & 1 & 1.4 \\
\hline ANCA & 185 & 93.9 & 8 & 4.3 & 115 & 92.7 & 4 & 3.5 & 70 & 95.9 & 4 & 5.7 \\
\hline C3 & 151 & 76.6 & 14 & 9.3 & 96 & 77.4 & 9 & 9.4 & 55 & 75.3 & 5 & 9.1 \\
\hline C4 & 151 & 76.6 & 5 & 3.3 & 96 & 77.4 & 4 & 4.2 & 55 & 75.3 & 1 & 1.8 \\
\hline anti-Ap-4 Abs & 83 & 42.1 & 0 & 0 & 52 & 41.9 & 0 & 0 & 31 & 42.5 & 0 & 0 \\
\hline HIV & 130 & 66 & 0 & 0 & 82 & 66.1 & 0 & 0 & 48 & 65.8 & 0 & 0 \\
\hline TPHA & 141 & 71.6 & 0 & 0 & 90 & 72.6 & 0 & 0 & 51 & 69.9 & 0 & 0 \\
\hline Antiphospholipid Abs & 38 & 19.3 & 1 & 2.6 & 27 & 21.8 & 1 & 3.7 & 11 & 15.1 & 0 & 0 \\
\hline HbA1c & 138 & 70.1 & 13 & 9.4 & 86 & 69.4 & 5 & 5.8 & 52 & 71.2 & 8 & 15.4 \\
\hline FABRY & 39 & 19.8 & 0 & 0 & 16 & 12.9 & 0 & 0 & 23 & 31.5 & 0 & 0 \\
\hline TSH & 155 & 78.7 & 7 & 4.5 & 99 & 79.8 & 1 & 1 & 56 & 76.7 & 6 & 10.7 \\
\hline Urine analysis & 94 & 47.7 & 3 & 3.2 & 63 & 50.8 & 3 & 4.8 & 31 & 42.5 & 0 & 0 \\
\hline
\end{tabular}

\section{Methods}

\section{Analysis of patient data}

This study is based on data from the records of patients treated at the Special Outpatient Clinic for Multiple Sclerosis of the Alfried Krupp Hospital Essen-Rüttenscheid in Essen, Germany.

The first step was to screen 554 patients referred between July 2010 and July 2014 with the diagnosis of "multiple sclerosis" or "suspected multiple sclerosis". Altogether 197 patients with MS or suspected MS according to the 2010 revised McDonald criteria who underwent the guideline-recommended laboratory tests during the differential diagnostic workup, with antinuclear antibody testing as the minimum requirement, were included in this study. Of these, 124 ultimately were diagnosed with definite MS. Since no particular course of MS was specified, patients with RRMS, SPMS and $\mathrm{CIS}$ were included in this study.

The approval of this study by the ethics committee of the University of Duisburg-Essen was available.

All data (laboratory tests (see - Table 1), CSF, MRI, evoked potentials, symptoms, comorbidities, family history) were retrospectively analyzed according to descriptive aspects based on the complete patient records in paper form and the electronic medical records.
The standardized laboratory tests and CSF examinations were carried either internally in the hospital laboratory or in an external laboratory. Evoked potentials were performed internally in the hospital using the same equipment. The standardized MRI scans were performed using various scanners located at different sites. MRI analysis was performed by a neuroradiology specialist, taking into account lesion load and increase in lesion load, configuration of lesions and contrast enhancement in axial and sagittal T2-, FLAIRand T1-weighted sequences with contrast. Symptoms, comorbidities and family history were documented by an experienced neurologist.

The results of these tests were compared in the total patient population with regard to abnormal and normal ANA titers, in the MS and non-MS patient populations.

\section{Questionnaire}

A subgroup of patients with ANA titers $\geq 1: 160$ was approached using a questionnaire (see $>$ Table 2 ) designed to obtain information about any in the meantime newly developed rheumatological symptoms and newly diagnosed conditions. These 38 patients were mostly contacted by phone and asked to prospectively answer these questions. 
- Table 2 Questions of the prospective survey.

1) Have you ever experienced redness and swelling of joints?
Yes（ )
No ( )

If yes, of which joint(s):

2) Have you ever experienced painful joints?

$\begin{array}{ll}\text { Yes ( ) No( ) } & \text { Not }\end{array}$

If yes, of which joint(s):

3) Have you ever had oral (aphthous) or genital ulcers?

Yes ( ) No( )

4) Have you ever experienced headaches?

Yes（） No（）

If yes, of what kind:

5) Have you ever had a stroke?

\begin{tabular}{l|l} 
Yes ( ) No( ) &
\end{tabular}

If yes, please provide details:

6) Have you ever had red spots on your lower legs/knees or ankles (erythema nodosum)?

Yes ( )

No ( )

7) Have you ever seen a rheumatologist to confirm/exclude a rheumatological disease?

Yes ( ) No( )

If yes, where:

8) Have you been diagnosed with a new disease in the meantime?

$\begin{array}{ll}\text { Yes ( ) No( ) } & \text { No }\end{array}$

If yes, which:

\section{Statistical analysis}

Statistical analysis was carried out using the Microsoft Excel for Mac 2011 software. For parametric methods, mean and median + /standard deviation were reported. The fourfold chi-square test for independence was used to determine statistical significance. A significance level of $p<0.05$ was used. ANA positivity was defined as any ANA titer $\geq 1: 80$ to increase sensitivity. Where the investigation of special questions required the use of a threshold titer of $\geq 1: 160$ to increase specificity, this was noted separately.

Given the exploratory nature of this study, no Bonferroni correction was performed as this could have obscured actually existing associations.

\section{Results}

\section{Demographic data}

The total sample size was 197 patients. Female predominance was found with a female:male ratio of 3:1 (146 (74.1\%) female and 51 (25.9\%) male patients).

Patient age at the time of initial presentation at the MS Outpatient Clinic ranged between 16 and 71 years (mean 41.4 years; median 42 years; SD 12.0), while patient age at the time of initial onset of symptoms was between 14 and 68 years (mean 36.7 ).

Altogether 124 of the 197 patients $(62.9 \%)$ had multiple sclerosis, including patients with CIS. Thirty patients (24.2\%) had CIS, 80 patients $(64.5 \%)$ relapsing-remitting MS and 14 patients
(11.3\%) secondary progressive MS. Accordingly, 73 of the 197 patients (37.1\%) had no MS.

MS patients did not differ with regard to age and gender from non-MS patients and from the total patient population.

\section{Laboratory tests}

- Table 1 reveals a rather low test rate. Test frequency was above $90 \%$ only for 5 (CRP, blood count, ANA, ANCA, ENA) of the mandatory parameters, indicating inconsistent implementation of the guideline recommendations.

Consequently, the following analyses focused on ANA titers because information about this parameter was available for all patients because of the ANA-based preselection and altogether $59.4 \%$ of these patients had positive ANA titers.

With regard of the other mandatory laboratory parameters, the total number of analyzed cases was considerably lower, but, most importantly, the frequency of abnormal results for these parameters was so low that no significance testing was performed.

\section{The total patient population}

\section{Positive ANA titers and rheumatologic diseases}

As shown in $\downarrow$ Table $1,59.4 \%$ of patients had positive ANA titers, while $40.6 \%$ were ANA-negative.

In the following, our analyses will focus on patients with positive ANA titers; marginally abnormal titers of $1: 80$ were also evaluated as positive. For positive ANA titers of $\geq 1: 160$, a subgroup analysis was performed.

Five of the 197 patients ( $2.5 \%$ ) of the total patient population had a rheumatologic disease ( 1 systemic lupus erythematosus, 1 ankylosing spondylitis, 1 psoriasis arthritis, 2 neuro-Behcet's disease). Four of these 5 patients were ANA-positive; consequently, 4 (3.4\%) of the altogether 117 ANA-positive patients had a rheumatologic disease. Two of these patients were additionally diagnosed with MS.

The finding of an increased ANA titer did not significantly distinguish between presence and absence of a rheumatologic disease $\left(\mathrm{p}<0.7 ; \mathrm{x}^{2}=0.239\right)$.

\section{Difference between patients with abnormal and normal laboratory parameters}

Tested and abnormal laboratory values are listed in \ Table 1.

In the total patient population, no statistically significant differences with regard to any of the tested laboratory parameters was found between presence and absence of MS (ANA titers 1:80 $(p=0.2)$, ANA titers $\geq 1: 160(p<0.6)$, ANCA $(p<0.8)$, dsDNA Abs $(p<0.7), C 3(p<0.9), C R P(<0.99), H b A 1 c(p<0.2)$. An exemption was oligoclonal banding which is positive in MS patients; this parameter differentiated between patients with and without MS on a high significance level $(p<0.0001)$, comparable with abnormal visually evoked potentials ( $p<0.0001)$. Here, $84 \%$ (100 of 119) of patients positive for oligoclonal bands had MS compared with $25 \%$ (13 of 52 ) of patients negative for oligoclonal bands.

\section{Results of the questionnaire analysis}

In 38 patients (19.3\%) of the total patient population, ANA titers were $\geq 1: 160$. Altogether 24 (63.2\%) of the 38 circulated questionnaires (see $>$ Table 2 ) were completed via mail or telephone. The 
mean interval between initial presentation and completion of the questionnaire was 1.9 years.

Sixteen of the patients $(66.7 \%)$ who completed the questionnaire had MS. Of these, 11 (68.8\%) were female and 5 (31.3\%) male.

Eight of the 24 patients (34.8\%) had no MS. Of these, 7 ( $87.5 \%$ ) were female and $1(12.5 \%)$ male.

Remarkably, despite elevated ANA titers none of the MS patients was diagnosed with a rheumatologic disease during the follow-up period and none of the non-MS patients developed MS.

\section{The MS patient population}

Positive ANA titers and rheumatologic diseases

As shown in - Table 1, $54.8 \%$ of MS patients (CIS $60 \%$; RRMS $52.5 \%$; SPMS $57.1 \%$ ) had positive ANA titers ( $\geq 1: 80$ ), while $45.2 \%$ were ANA-negative.

Assuming a threshold for ANA positivity of $\geq 1: 160,26.7 \%$ of CIS patients, $13.8 \%$ of RRMS patients and $21.4 \%$ of SPMS patients tested positive for ANA.

Two (1.6\%) of the MS patients with ANA titers of 1:80 had a rheumatological disease (psoriasis arthritis and ankylosing spondylitis) in addition to MS.

\section{Differences between MS patients with and without ANA titers}

Tested and abnormal laboratory values are listed in $>$ Table $\mathbf{1}$.

In $13.2 \%$ of the ANA-positive (titer $1: 80$ ) MS patients, an additional autoimmune disease was found, while this was the case in only $1.8 \%$ of the ANA-negative patients. This difference was significant $(p<0.05)$. Apart from the above mentioned rheumatologic diseases, autoimmune conditions included Hashimoto's disease, (5), psoriasis (1), uveitis (2), and primary biliary cholangitis (1). However, for ANA-positive MS patients with titers $\geq 1: 160$ this difference was not significant $(p<0.6)$.

The comparison of ANA-positive and ANA-negative MS patients with regard to RRMS and SPMS types found no significant differences $\left(p<0.7 ; x^{2}=0.267\right.$ and $p<0.95 ; x^{2}=0.01$, respectively).

ANA-positive MS patients did not differ significantly from ANA-negative MS patients with regard to evoked potentials (EP) (VEP $(p<0.3)$; tibial nerve SEP $(p=0.975)$; median nerve SEP $(p<0.95))$ and MRI parameters ( $\geq 2$ lesions $(p<0.5)$; periventricular lesions ( $p<0.8)$; relation to corpus callosum $(p<0.95)$; contrast enhancement $(p<0.975)$; increase in lesion load $(p<0.2))$. The same applied to clinical symptoms (sensory symptoms $(p<0.95)$; motor symptoms ( $p<0.05)$; ataxia $(p<0.6)$; gait abnormalities $(p<0.8)$; Lhermitte's sign ( $p<0.9)$; cranial nerve involvement, including RBN ( $p<0.8)$; urge symptoms ( $p<0.975)$, and the presence of oligoclonal bands $(p<0.3)$.

While no association was found between ANA positivity and myocardial infarction $(p<0.3)$, autoimmune disease $(p<0.2)$ and family history of psychiatric disease ( $p<0.5), 17.6 \%$ of ANA-positive (titer 1:80) MS patients had a positive family history for MS. By contrast, in the group of ANA-negative MS patients, this applied to none of the patients-a highly significant difference $(p<0.005)$. However, for ANA-positive MS patients with titers $\geq 1: 160$, the result was non-significant $(p<0.2)$.

\section{The non-MS patient population}

\section{Differential diagnosis of multiple sclerosis}

The 73 patients without findings indicative of multiple sclerosis were classified in five distinct differential diagnostic groups:

- Group 1: unspecific white matter lesions (24 patients; 32.9\%)

- Group 2: psychiatric/psychosomatic conditions (14 patients; $19.2 \%)$

- Group 3: isolated myelitis (10 patients; $13.7 \%$ )

- Group 4: other/rare diagnoses (21 patients; $28.8 \%$ )

- Group 5: of unclear etiology (4 patients; $5.5 \%$ ) Group 4 comprised:

- Small-fiber neuropathy

- Suspected migraine with aura

- Suspected lesion of right superficial peroneal nerve

- Pituitary space-occupying lesion with hemorrhage

- Cerebral microangiopathy

- Suspected asymmetrical axonal PNP

- Paraneoplastic neuromuscular syndrome along with multiple myeloma

- Vitamine B12 deficiency-induced sensory loss

- CADASIL

- Status post brainstem encephalitis

\section{Positive ANA titers and rheumatologic diseases}

As shown in $>$ Table 1, 67.1\% of patients without MS had positive ANA titers, while $32.9 \%$ of these patients were ANA-negative.

Based on their medical history, previous findings and MRI scans, 3 patients (4.1\%) were diagnosed with rheumatologic conditions (2 neuro-Behcet's disease and 1 systemic lupus erythematosus). Two of these 3 patients (66.7\%) were ANA-positive and 1 patient (33.3\%) ANA-negative.

\section{Differences between non-MS patients with and without ANA titers}

Tested and abnormal laboratory values are listed in > Table $\mathbf{1}$.

In the non-MS patient group, no significant differences were found regarding positive or negative ANA titers and headache $\left(p<0.5 ; x^{2}=0.54\right)$, aphthous ulcers $\left(p<0.3 ; x^{2}=1.272\right)$, arthralgia $\left(p<0.9 ; x^{2}=0.028\right)$ and pain $\left(p<0.6 ; x^{2}=0.412\right)$, the secondary diagnosis of migraine $\left(p<0.3 ; x^{2}=1.344\right)$ and the presence of oligoclonal bands $\left(p<0.9 ; x^{2}=0.020\right)$.

However, $59.2 \%$ of ANA-positive non-MS patients had a secondary psychiatric diagnosis, while this was the case in none of the ANA-negative non-MS patients. This difference was highly significant $\left(p<0.0001 ; x^{2}=21.159\right)$.

For positive or negative ANA titers and MS-positive family history $\left(\mathrm{p}<0.9 ; \mathrm{x}^{2}=0.024\right)$, stroke $\left(\mathrm{p}<0.95 ; \mathrm{x}^{2}=0.007\right)$ and autoimmune disease $\left(p<0.9 ; x^{2}=0.024\right)$, no significant differences were found.

Correlations of ANA status with assignment to the various differential diagnostic groups (groups 1-5) were not statistically significant (group 1/ white matter lesions: $p<0.1 ; x^{2}=3.23$; group 2 | psychiatric conditions: $p<0.1 ; x^{2}=3.362$; group $3 /$ isolated myelitis: $p<0.9 ; x^{2}=0.024$; group 4/other diagnoses: $p<0.9 ; x^{2}=0.049$; group 5 was not analyzed as sample size was too small). However, there was a trend towards a higher prevalence of white matter lesions in ANA-positive non-MS patients compared with ANA-nega- 
tive non-MS patients $(40.8 \%$ and $16,7 \%$, respectively $[p<0,1$; $\left.\left.x^{2}=3,23\right]\right)$.

In addition, the fourfold chi-square test for independence was performed to evaluate the earlier significant result regrading psychiatric secondary diagnoses in patients with ANA titers of $\geq 1: 160$. Here, the differences between ANA-positive and ANA-negative patients were no longer significant $\left(p<0.5 ; x^{2}=0.461\right)$

\section{Discussion}

Wilhelm of Ockham (1288-1347), a medieval philosopher, theologian and ecclesiastical writer, postulated the principle known as Occam's razor, stating that in case of several competing explanations for the same observation, the simplest theory should be selected.

Applied to clinical daily practice, it means that when several potential explanations for a set of clinical signs and symptoms are available, the one with the fewest assumptions should be selected, representing the "simplest theory".

Thus, with regard to MS the question arises whether in patients presenting with fairly typical clinical features of MS each and every condition listed in the possible differential diagnosis of the disease should be ruled out using laboratory testing and diagnostic technologies to identify, for example, a case of otherwise misinterpreted neuropsychiatric systemic lupus erythematosus or neuro-Behçet's disease, or whether to assume the patient has MS, a common disease, is more appropriate in case patient age, medical history as well as physical examination and MRI findings are in line with those characteristic for MS.

However, an online survey of 122 MS specialists performed in 2012 revealed that MS is a common misdiagnosis [17]. As many as $95.1 \%$ of respondents stated to have treated at least one patient misdiagnosed with MS in the last year. Nonspecific white matter lesions, microangiopathic changes, migraine, psychiatric illness, and NMO spectrum diseases were reported as the most common misdiagnoses. As causes of misdiagnosis, uncritical adoption of radiological evaluations and inaccurate interpretation of findings were named. Misdiagnosis had negative health implications for patients and increased healthcare costs [17].

However, an uncritical "diagnostic watering can" approach without defined stepwise diagnostic processes also results in additional costs to the healthcare system and confusion among patients. Apart from further costs arising from additional visits to rheumatologists, the total costs for mandatory and facultative laboratory parameters amount to approximately EUR 361, of which about EUR 169 are attributed to mandatory tests and about EUR 192 to facultative laboratory parameters.

As part of the Choosing Wisely campaign, the Canadian Rheumatology Association issued the following recommendations: “Don't order ANA as a screening test in patients without specific signs or symptoms of systemic lupus erythematosus (SLE) or another connective tissue disease (CTD)“. [18-19]

The US-American Choosing Wisely recommendations did not go quite so far, but stated clearly: “Don't test ANA sub-serologies without a positive ANA and clinical suspicion of immune-mediated disease“. [20-22]
This stepwise diagnostic approach starts with screening for antinuclear antibodies using immunofluorescence (IFT). Further testing, e. g., with anti-ENA profile or anti-dsDNA antibodies, should only be undertaken if indicated by titers and fluorescence patterns [23]. In the guidelines, this stepwise diagnostic approach is not used for anti-dsDNA antibodies as these are included in the mandatory laboratory parameter category, together with ANA.

However, the likelihood of positive anti-dsDNA or anti-ENA findings is influenced by the level of the ANA titer-typically, determining anti-dsDNA or anti-ENA antibodies is only of diagnostic value with ANA titers $\geq 1: 320$.

Our study showed that ANA and the other analyzed laboratory parameters, such as anti-ENA, anti-ANCA, anti-dsDNA antibodies or CRP, failed to differentiate between the presence and absence of MS and were not predictive of the presence of rheumatological disease. By contrast, positive oligoclonal bands and abnormal visual evoked potentials (VEPs) were able to distinguish between MS and its differential diagnosis on a high significance level. The predictive value of oligoclonal bands for developing MS is well established: for example, $50 \%$ of patients with positive oligoclonal bands developed overt MS after approximately 4 years, but only $16 \%$ of patients with negative oligoclonal bands [24].

The lack of difference between the MS group and non-MS group with regard to the rate of positive ANA titers is best explained by the fact that increased ANA titers are also found in healthy persons.

Tan et al. reported in 1997 that low ANA titers of 1:40 occur in up to $30 \%$ of healthy individuals and ANA titers of 1:80, 1:160 and $1: 320$ in $13.3 \%, 5 \%$ and up to $3.3 \%$ of healthy persons, respectively [25]. In 2000, Vaile et al. published their finding that high titers of autoantibodies, while being indicative of disease, are not necessarily indicative of a connective tissue disorder. These auto-antibodies are found in patients with other diseases and in clinically healthy individuals as well [25-27].

A positive family history of MS is in MS patients significantly associated with positive ANA titers at the 1:80 level which may indicate a genetic predisposition for MS, presenting as an autoimmune dysregulation. However, we were not able to reproduce this result when we used an ANA titer of 1:160 as the cut-off value.

Already in 1995, Barned et al. stated that increased ANA titers in MS are best explained by autoimmune dysregulation [13]. Two years later, this view was confirmed by Collard et al. [14]. In their study conducted in 1997, $22.5 \%$ of MS patients had positive ANA titers, independent of gender, age, MS duration or course and disability [14].

Our study also indicates that an unspecific autoimmune disposition is reflected in the borderline titer range of $1: 80$, especially in the light of the lack of significant differences when a cut-off titer of 1:160 was used.

Likewise, using a cut-off titer of 1:160, it was not possible to reproduce the significant associations with MS found for a cut-off titer of 1:80 in patients with other concomitant autoimmune disease, such as Hashimoto's disease, uveitis and primary biliary cholangitis. Earlier, various studies had indicated an increased prevalence of autoimmune disease in MS patients and their biological relatives $[28,29]$. However, after adjustment for age and gender, the results did not confirm an increased occurrence of autoimmune disease among MS patients or their relatives [30]. 
The authors of an Argentine study conducted in 2014 found no increased risk for other autoimmune disease in MS patients and no increased risk to develop MS if another autoimmune disease was present [31]. This study did not differentiate between ANA positivity and ANA negativity.

Likewise, in our study we found no association between positive ANA titers and specific clinical symptoms or constellations of findings which would be suggestive of the presence of ANA.

In a second step, we followed the clinical course of subjects with an ANA titer > 1:160 prospectively over a period of 1.9 years, using a questionnaire, to increase the power of this retrospective study.

During this period, no rheumatologic disease was diagnosed in the MS patient group.

In the group of patients without MS or a rheumatologic disease, no case of MS was observed after a period of almost 2 years. However, about half of the patients in the non-MS group were diagnosed with another chronic disease in the meantime, including 1 patient with CADASIL, 1 with ALS and mixed connective-tissue disease, 1 with fibromyalgia and 2 with rheumatologic diseases.

However, it should be noted that in the prospective part of our study only 24 patients were followed up. As a result, the number of patients in each group was too low to allow testing of statistical significance of differences.

Other limitations of our study include potential selection bias and recruitment bias since all patients who participated in this study had been referred to a special outpatient clinic for multiple sclerosis. For example, a higher proportion of differential diagnoses than actually found in this study would have been expected. The sample size of just about 200 patients may have been too small given the rarity of conditions such as neuropsychiatric systemic lupus erythematosus und especially Fabry's disease. In addition, comparisons with the general population were not possible due to the pre-selected nature of the study population.

As another potential source of bias, the low analysis rates have to be taken into account ( $\vee$ Table 1 ). In addition, it should be noted that the timing of sampling for laboratory testing was not uniform in relation to the first manifestation of multiple sclerosis.

A positive aspect of the study is that it covered a wide range of evaluated parameters, enabling comprehensive comparisons of clinical symptoms, secondary diagnoses, family history, MRI findings, laboratory parameters, and evoked potentials.

However, the results of this study are to be viewed as preliminary since no Bonferroni correction was performed given its explorative nature. They need to be confirmed by a hypothesis-driven and powered larger study.

In summary, the results of this study indicate that uncritical mandatory and broad autoantibody testing, especially of antinuclear antibodies (ANA), may not be helpful in the differential-diagnostic workup of multiple sclerosis [5].

Without clinical signs of a connective tissue disorder, a positive ANA test result is irrelevant and only leads to unnecessary additional investigations, delayed diagnosis, patient distress or even psychosomatic fixation.

In our opinion, at the time of first diagnosis of MS it is sufficient to take a detailed medical history (family history? previous symptoms? aphthous ulcers? arthralgia?) and perform a thorough, not only neurological but also general physical examination (erythema nodosum? angiokeratoma? livedo racemosa?) in combination with the interpretation of MRI scans and cerebral spinal fluid findings to rule out conditions included in the differential diagnosis of MS.

Thus, we would recommend in line with the rheumatologic Canadian Choosing Wisely recommendations to refrain from the analysis of mandatory autoimmune laboratory parameters [18]. Examiners who cannot decide for this approach should at least take into account the US Choosing Wisely recommendations and refrain from sub-differentiation in patients with unremarkable ANA titers [20].

\section{Acknowledgement}

We would like to extent our sincere thanks Yuriko Stiegler, M.D., Specialist in Laboratory Medicine.

\section{References}

[1] Polman CH, Reingold SC, Banwell B et al. Diagnostic criteria for multiple sclerosis: 2010 revisions to the McDonald criteria. Ann Neurol 2011; 69: 292-302

[2] Swanton JK, Rovira A, Tintore M et al. MRI criteria for multiple sclerosis in patients presenting with clinically isolated syndromes: A multicentre retrospective study. Lancet Neurol 2007; 6: 677-686

[3] Kollia K, Maderwald S, Putzki $\mathrm{N}$ et al. First clinical study on ultra-highfield MR imaging in patients with multiple sclerosis: Comparison of 1.5T and 7T. AJNR Am J Neuroradiol 2009; 30: 699-702

[4] Dixon JE, Simpson A, Mistry N et al. Optimisation of $\mathrm{T}(2)^{*}$-weighted MRI for the detection of small veins in multiple sclerosis at $3 \mathrm{~T}$ and $7 \mathrm{~T}$. Eur J Radiol 2013; 82: 719-727

[5] Gold R, Hemmer B, Wiendl H. Leitlinie Diagnose und Therapie der Multiplen Sklerose. In: Diener HC, Weimar C, (Hrsg.). Leitlinien für Diagnostik und Therapie in der Neurologie. Stuttgart: Thieme Verlag; 2014

[6] Arinuma Y, Kikuchi $\mathrm{H}$, Wada T et al. Brain MRI in patients with diffuse psychiatric/neuropsychological syndromes in systemic lupus erythematosus. Lupus Sci Med 2014; 1: e000050

[7] Saruhan-Direskeneli G, Yentur SP, Mutlu M et al. Intrathecal oligoclonal $\mathrm{IgG}$ bands are infrequently found in neuro-Behcet's disease. Clin Exp Rheumatol 2013; 31: 25-27

[8] Chabriat H, Joutel A, Dichgans M et al. Cadasil. Lancet Neurol 2009; 8: 643-653

[9] Bottcher T, Rolfs A, Tanislav C et al. Fabry disease - underestimated in the differential diagnosis of multiple sclerosis? PLoS One 2013; 8: e71894

[10] Becker J, Rolfs A, Karabul N et al. D313Y mutation in the differential diagnosis of white matter lesions: Experiences from a multiple sclerosis outpatient clinic. Mult Scler 2016; 22: 1502-1505

[11] Karussis D, Leker RR, Ashkenazi A et al. A subgroup of multiple sclerosis patients with anticardiolipin antibodies and unusual clinical manifestations: do they represent a new nosological entity? Ann Neurol 1998; 44: 629-634

[12] Heinzlef O, Weill B, Johanet C et al. Anticardiolipin antibodies in patients with multiple sclerosis do not represent a subgroup of patients according to clinical, familial, and biological characteristics. J Neurol Neurosurg Psychiatry 2002; 72: 647-649

[13] Barned S, Goodman AD, Mattson DH. Frequency of anti-nuclear antibodies in multiple sclerosis. Neurology 1995; 45: 384-385

[14] Collard RC, Koehler RP, Mattson DH. Frequency and significance of antinuclear antibodies in multiple sclerosis. Neurology 1997; 49: 857-861 
[15] Garg N, Zivadinov R, Ramanathan M et al. Clinical and MRI correlates of autoreactive antibodies in multiple sclerosis patients. J Neuroimmunol 2007; 187: 159-165

[16] Etemadifar M, Fatemi A, Hashemijazi $\mathrm{H}$ et al. Is it necessary to perform connective tissue disorders laboratory tests when a patient experiences the first demyelinating attack? J Res Med Sci 2013; 18: 617-620

[17] Solomon AJ, Klein EP, Bourdette D. "Undiagnosing" multiple sclerosis: The challenge of misdiagnosis in MS. Neurology 2012; 78: 1986-1991

[18] Chow SL, Carter Thorne J, Bell M] et al. Choosing wisely: The Canadian Rheumatology Association's list of 5 items physicians and patients should question. J Rheumatol 2015; 42: 682-689

[19] Ferrari R. Evaluation of the Canadian Rheumatology Association Choosing Wisely recommendation concerning anti-nuclear antibody (ANA) testing. Clin Rheumatol 2015; 34: 1551-1556

[20] Rouster-Stevens KA, Ardoin SP, Cooper AM et al. Choosing Wisely: The American College of Rheumatology's Top 5 for pediatric rheumatology. Arthritis Care Res (Hoboken) 2014; 66: 649-657

[21] Kavanaugh A, Tomar R, Reveille J et al. Guidelines for clinical use of the antinuclear antibody test and tests for specific autoantibodies to nuclear antigens. American College of Pathologists. Arch Pathol Lab Med 2000; 124: 71-81

[22] Fritzler M]. Choosing wisely: Review and commentary on anti-nuclear antibody (ANA) testing. Autoimmun Rev 2016; 15: 272-280
[23] Verstegen G, Duyck MC, Meeus P et al. Detection and identification of antinuclear antibodies (ANA) in a large community hospital. Acta Clin Belg 2009; 64: 317-323

[24] Tintore M, Rovira A, Rio J et al. Do oligoclonal bands add information to MRI in first attacks of multiple sclerosis? Neurology 2008; 70 : 1079-1083

[25] Tan EM, Feltkamp TE, Smolen JS et al. Range of antinuclear antibodies in "healthy" individuals. Arthritis Rheum 1997; 40: 1601-1611

[26] Vaile JH, Dyke L, Kherani R et al. Is high titre ANA specific for connective tissue disease? Clin Exp Rheumatol 2000; 18: 433-438

[27] Illei GG, Klippel JH. Why is the ANA result positive? Bull Rheum Dis 1999; 48: 1-4

[28] Barcellos LF, Kamdar BB, Ramsay PP et al. Clustering of autoimmune diseases in families with a high-risk for multiple sclerosis: a descriptive study. Lancet Neurol 2006; 5: 924-931

[29] Broadley SA, Deans J, Sawcer S] et al. Autoimmune disease in first-degree relatives of patients with multiple sclerosis. A UK survey. Brain 2000; 123 (Pt 6): 1102-1111

[30] Ramagopalan SV, Dyment DA, Valdar W et al. Autoimmune disease in families with multiple sclerosis: a population-based study. Lancet Neurol 2007; 6: 604-610

[31] Farez MF, Balbuena Aguirre ME, Varela F et al. Autoimmune disease prevalence in a multiple sclerosis cohort in Argentina. Mult Scler Int 2014; 2014: 828162 